\title{
Microarray Analysis of the Molecular Mechanism Involved in Parkinson's Disease
}

\author{
Cheng Tan, Xiaoyang Liu, and Jiajun Chen \\ Department of Neurology, China-Japan Union Hospital of Jilin University, Changchun, Jilin 130033, China \\ Correspondence should be addressed to Jiajun Chen; cjj@jlu.edu.cn
}

Received 24 May 2017; Revised 21 August 2017; Accepted 18 October 2017; Published 1 March 2018

Academic Editor: Amnon Sintov

Copyright (c) 2018 Cheng Tan et al. This is an open access article distributed under the Creative Commons Attribution License, which permits unrestricted use, distribution, and reproduction in any medium, provided the original work is properly cited.

Purpose. This study aimed to investigate the underlying molecular mechanisms of Parkinson's disease (PD) by bioinformatics. Methods. Using the microarray dataset GSE72267 from the Gene Expression Omnibus database, which included 40 blood samples from PD patients and 19 matched controls, differentially expressed genes (DEGs) were identified after data preprocessing, followed by Gene Ontology (GO) and Kyoto Encyclopedia of Genes and Genomes (KEGG) pathway enrichment analyses. Protein-protein interaction (PPI) network, microRNA- (miRNA-) target regulatory network, and transcription factor- (TF-) target regulatory networks were constructed. Results. Of 819 DEGs obtained, 359 were upregulated and 460 were downregulated. Two GO terms, "rRNA processing" and "cytoplasm," and two KEGG pathways, "metabolic pathways" and "TNF signaling pathway," played roles in PD development. Intercellular adhesion molecule 1 (ICAM1) was the hub node in the PPI network; hsamiR-7-5p, hsa-miR-433-3p, and hsa-miR-133b participated in PD pathogenesis. Six TFs, including zinc finger and BTB domaincontaining 7A, ovo-like transcriptional repressor 1, GATA-binding protein 3, transcription factor dp-1, SMAD family member 1, and quiescin sulfhydryl oxidase 1, were related to PD. Conclusions. "rRNA processing," "cytoplasm," "metabolic pathways," and "TNF signaling pathway" were key pathways involved in PD. ICAM1, hsa-miR-7-5p, hsa-miR-433-3p, hsa-miR-133b, and the abovementioned six TFs might play important roles in PD development.

\section{Introduction}

Parkinson's disease (PD) is one of the most common agerelated neurodegenerative diseases [1]. The age at $\mathrm{PD}$ onset is approximately 55 years, and the incidence in the population aged $>65$ years is approximately $1 \%$ [1-3]. PD mainly occurs because of the death of dopaminergic neurons in the substantia nigra [4]. Patients with PD present with symptoms such as bradykinesia, resting tremor, rigidity, and postural instability [5]. The current therapy for PD is targeted at its symptoms rather than at dopaminergic neuron degeneration [1]. The diagnosis of PD at the early stage is challenging, and successfully managing PD is difficult at its later stages [4]. To date, the cause of PD remains unknown; however, it appears to involve the intricate interplay of environmental and genetic factors $[1,4]$.

Much effort has been spent in investigating PD pathogenesis, and the misfolding, aggregation, and aberrance of proteins are considered to be some of the main causes
$[1,4,5]$. Some key genes such as hydrogen sulfide, chromobox 5 (CBX5), and transcription factor 3 (TCF3) are related to PD $[6,7]$. Several pathways have also been identified to be related to $\mathrm{PD}$. Activation of the protein kinase B (Akt)/glycogen synthase kinase 3 beta/(GSK3 $\beta$ ) pathway by urate reportedly protects dopaminergic neurons in a rat model of PD [8]. In addition, the E2-related factor 2 (Nrf2)/antioxidant response element pathway reportedly counteracts mitochondrial dysfunction, which is a prominent PD feature [9]. The ubiquitin, lipid, nigrostriatal, autophagy-lysosome, and endosomal pathways are also involved in PD [10-15]. Furthermore, a recent study revealed several microRNAs (miRNAs) associated with PD; miR-205 suppresses LRRK2 expression and miR-205 expression levels in the brains of patients with PD decreases [16]. Furthermore, miR-34b and miR-34c are downregulated in the brains of patients with $\mathrm{PD}$, which is related to the reduction in the expression of DJ-1 and PARKIN [17], and miR-133 and miR-7 are also associated with PD [18-20]. Numerous 


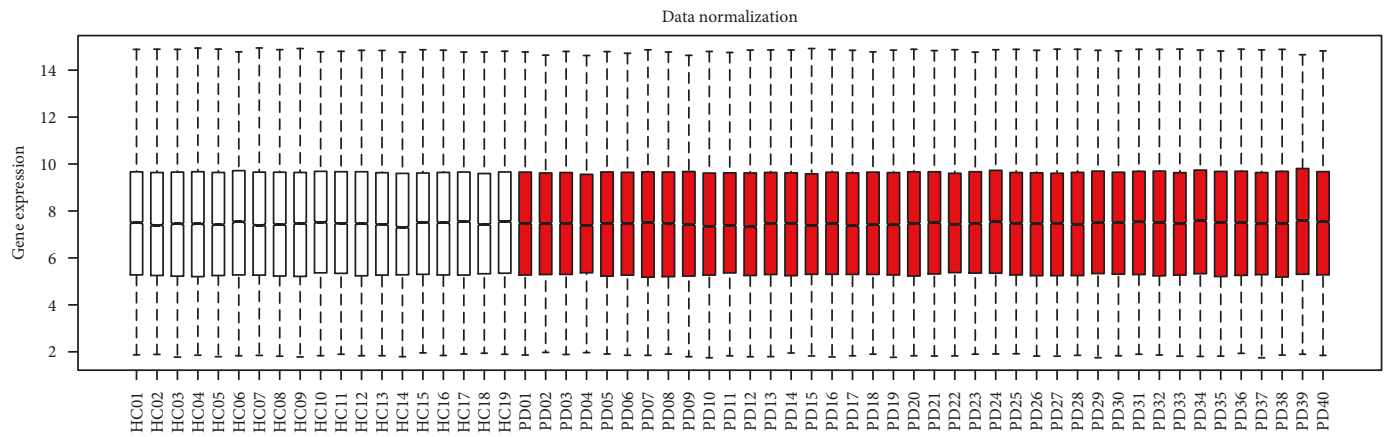

FIGURE 1: Boxplots for normalized gene expression data. Red represents the blood samples of patients with Parkinson's disease, and white represents the healthy matched control samples.

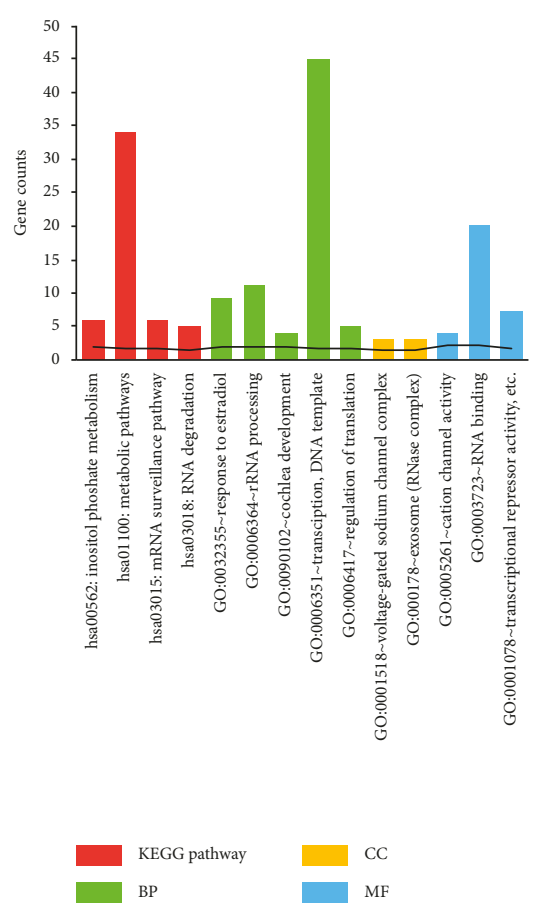

(a)

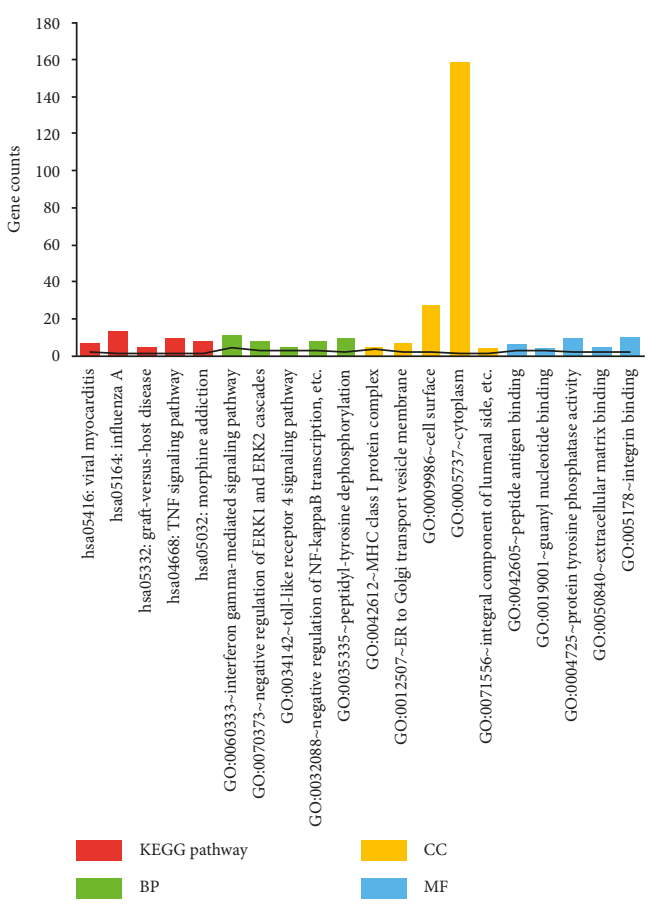

(b)

Figure 2: Functional enrichment analyses of differentially expressed genes (DEGs). (a) Gene Ontology (GO) terms and the Kyoto Encyclopedia of Genes and Genomes (KEGG) pathways of upregulated DEGs and (b) GO terms and KEGG pathways of downregulated DEGs. The numbers on the $x$-axis were the ID of pathways or GO terms. The numbers on the $y$-axis were gene counts.

reports that have described the roles of transcription factors (TFs) in PD have also been published. The TF paired-like homeodomain 3 has roles in developing and maintaining dopaminergic neurons [21, 22], and engrailed 1 , which is downregulated in the rat models, plays a role in the apoptosis of dopaminergic neurons and the symptoms of $\mathrm{PD}$ [23]. Moreover, Nrf2, nuclear factor kappa B (NF- $\kappa \mathrm{B})$, GATA2, and PHD finger protein 10 are TFs involved in PD [24-27]. However, understanding the key mechanisms underlying the development of PD remains unclear.

In a previous study, the microarray dataset GSE72267 generated by Calligaris et al. [7] was used to identify key differentially expressed genes (DEGs) such as CBX5, TCF3, dedicator of cytokinesis 10, and mannosidase alpha class 1C in the blood of patients with PD compared with those of healthy controls. Moreover, crucial pathways related to chromatin remodeling and methylation were revealed. In the current study, we downloaded this microarray dataset to comprehensively analyze DEGs in patients with PD compared with those in matched controls by bioinformatics approaches and to describe their functional annotations. Compared with the previous analysis conducted by Calligaris et al. [7], we performed additional analyses, including those for the protein-protein interaction (PPI), miRNAtarget regulatory, and TF-target regulatory networks, to further elucidate the key mechanisms underlying PD. Our 


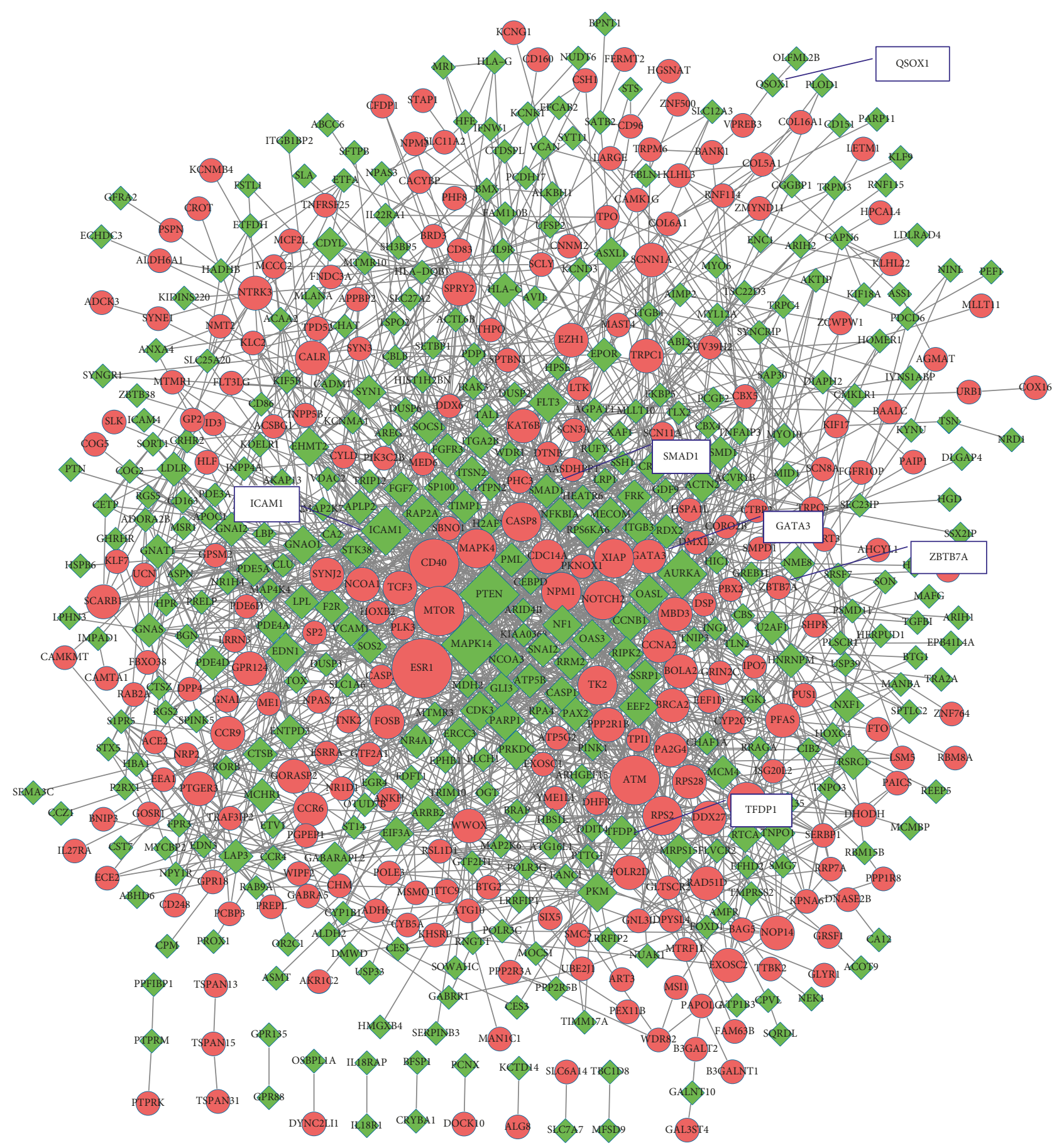

FIGURE 3: The protein-protein interaction (PPI) network of differentially expressed genes (DEGs). Red circles represent upregulated DEGs, and green diamonds represent downregulated DEGs.

results may provide useful data for diagnosing and treating PD.

\section{Materials and Methods}

2.1. Affymetrix Microarray Data. Gene expression profile data GSE72267 was extracted from the Gene Expression Omnibus database (https://www.ncbi.nlm.nih.gov/geo/) [28]. The GSE72267 dataset was deposited by Calligaris et al. [7], including blood samples from 40 PD patients and 19 healthy matched controls and was based on the platform of the GPL571 (HG-U133A-2) Affymetrix Human Genome U133A 2.0 Array (Affymetrix Inc., Santa Clara, California, USA). This dataset was downloaded and analyzed on October 2016.

2.2. Data Preprocessing and DEG Screening. The downloaded data in CEL files were preprocessed using the Affy package 
TABLE 1: List of top 10 differentially expressed genes with higher degrees in protein-protein interaction network.

\begin{tabular}{|c|c|c|c|}
\hline Gene & Full name & Description & Degree \\
\hline MAPK14 & Mitogen-activated protein kinase 14 & Down & 68 \\
\hline ESR1 & Estrogen receptor 1 & Up & 54 \\
\hline PTEN & Phosphatase and tensin homolog & Down & 52 \\
\hline MTOR & Mechanistic target of rapamycin & Up & 40 \\
\hline ATM & ATM serine/threonine kinase & Up & 35 \\
\hline ICAM1 & Intercellular adhesion molecule 1 & Down & 33 \\
\hline $\mathrm{CD} 40$ & CD40 molecule & Up & 32 \\
\hline AURKA & Aurora kinase A & Down & 31 \\
\hline PRKDC & Protein kinase, DNA-activated, catalytic polypeptide & Down & 29 \\
\hline TK2 & Thymidine kinase 2 , mitochondrial & Up & 29 \\
\hline
\end{tabular}

Degree was used for describing the importance of protein nodes in network. The higher the degree was, the more important the nodes were in network.

(version 1.50.0) [29] in R language, including background correction, normalization, and expression calculation. Annotations to the probes were performed, and probes that were not matched to the gene symbol were excluded. The average expression values were taken if different probes mapped to the same gene. DEGs in patients with PD compared with those in healthy matched controls were analyzed using the limma package (version 3.10.3) [30] in $\mathrm{R}$ language. The cutoff threshold was set to a $p$ value of $<0.05$.

2.3. Pathway Enrichment Analysis. Gene ontology (GO) (http://www.geneontology.org/) analysis is commonly used for functional studies of large-scale genomic or transcriptomic data and classifies functions with respect to three aspects: molecular function (MF), cellular component (CC), and biological process (BP) [31, 32]. The Kyoto Encyclopedia of Genes and Genomes (KEGG; http://www.kegg.jp/) pathway database [33] is widely used for systematic analysis of gene functions, linking genomic data with higher order functional data. The database for annotation, visualization, and integrated discovery (DAVID) is an integrated biological knowledgebase with analytical tools used for systematic and integrative analysis of large gene lists [34]. In this study, GO terms and KEGG pathway enrichment analyses for up- and downregulated DEGs were performed using DAVID (version 6.8). The cutoff thresholds were as follows: an enrichment gene number count of $\geq 2$ and a super geometry inspection significance threshold $p$ value of $<0.05$.

2.4. PPI Network Analysis. Search Tool for the Retrieval of Interacting Genes/Proteins (STRING; http://www.string-db. org/) [35] is an online database that assesses and integrates PPIs. In this study, DEGs were mapped into the STRING database for PPI analysis, with a PPI score of 0.4 as the parameter setting. The PPI network established by DEGs was constructed using the Cytoscape software (version 3.2.0) [36], and the topology scores of the nodes, including node degree in the PPI network, were analyzed using the CytoNCA plugin (version 2.1.6; http://apps.cytoscape. org/apps/cytonca) [37] (parameter setting: without weight). Degree was used for describing importance of protein nodes in network. The higher the degree was, the more important the nodes were in network. In addition, subnetworks were identified using the MCODE plugin [38] in the Cytoscape software, and subnetworks with a score of $>5$ were identified as key subnetworks. Finally, KEGG pathway enrichment analyses for the genes in the key subnetworks were performed.

2.5. miRNA-Target Regulatory Network Analysis. The miR2disease (http://www.mir2disease.org/) database [39] is a manually curated database that provides a comprehensive resource of miRNA deregulation in various human diseases. miRWalk2.0 (http://zmf.umm. uni-heidelberg.de/apps/zmf/mirwalk2/) [40] is a comprehensive database that presents predicted and validated data, regarding miRNA targets in human, mouse, and rats. In this study, miRNAs related to PD were extracted from the miR2disease database, and experimentally verified miRNA-gene regulatory pairs were obtained by searching miRWalk2.0. Finally, a miRNA-target regulatory network was constructed by comparing DEGs with obtained miRNA-gene regulatory pairs using the Cytoscape software.

2.6. TF-Target Regulatory Network Analysis. The genes in the PPI network described above were further analyzed to identify TF-target interaction pairs that were then used to construct a TF-target regulatory network. The iRegulon plugin (version 1.3; http://apps.cytoscape.org/apps/iRegulon) [41] in the Cytoscape software collects multiple human TF-target interaction databases such as Transfac, Jaspar, and Encode using two computational methods: Motif and Track. In this study, we analyzed the TF-target pairs using the iRegulon plugin and compared them with TFs with DEGs in the PPI network, followed by a TF-target regulatory network construction. The parameter settings were as follows: minimum identity between orthologous genes, 0.05 and maximum false discovery rate on motif similarity, 0.001 . The normalized enrichment score (NES) indicates the reliability of the results, and the cutoff threshold was NES of $>3$. 


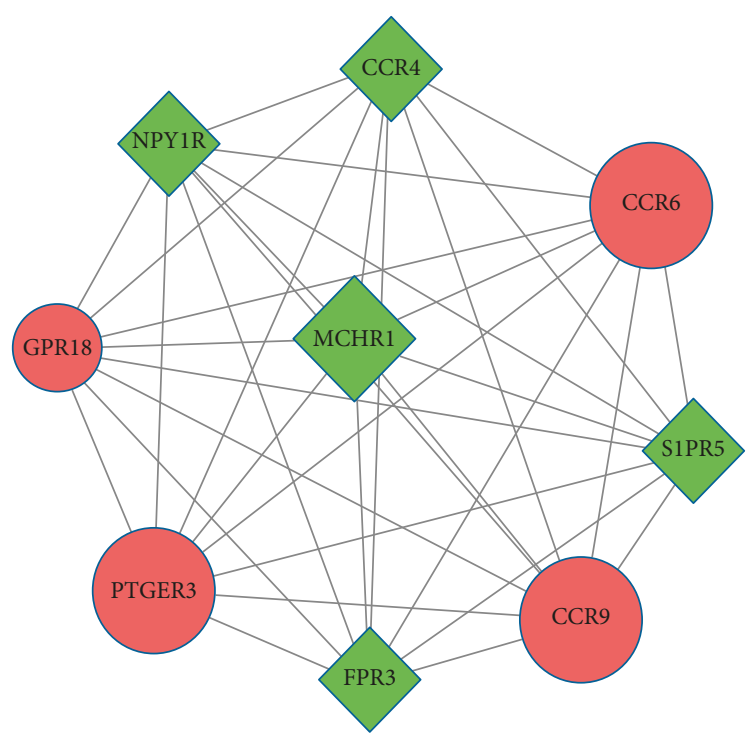

(a)

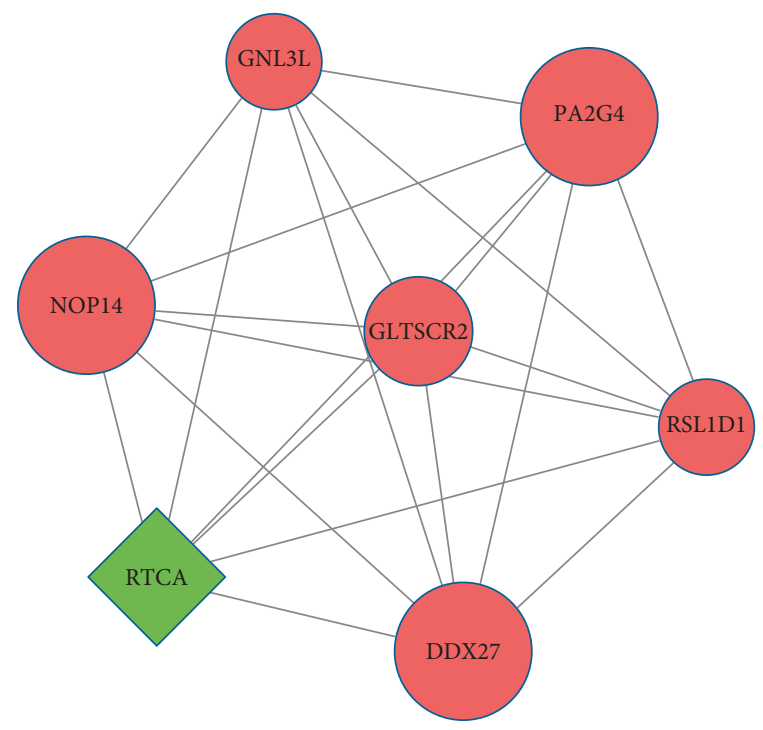

(b)

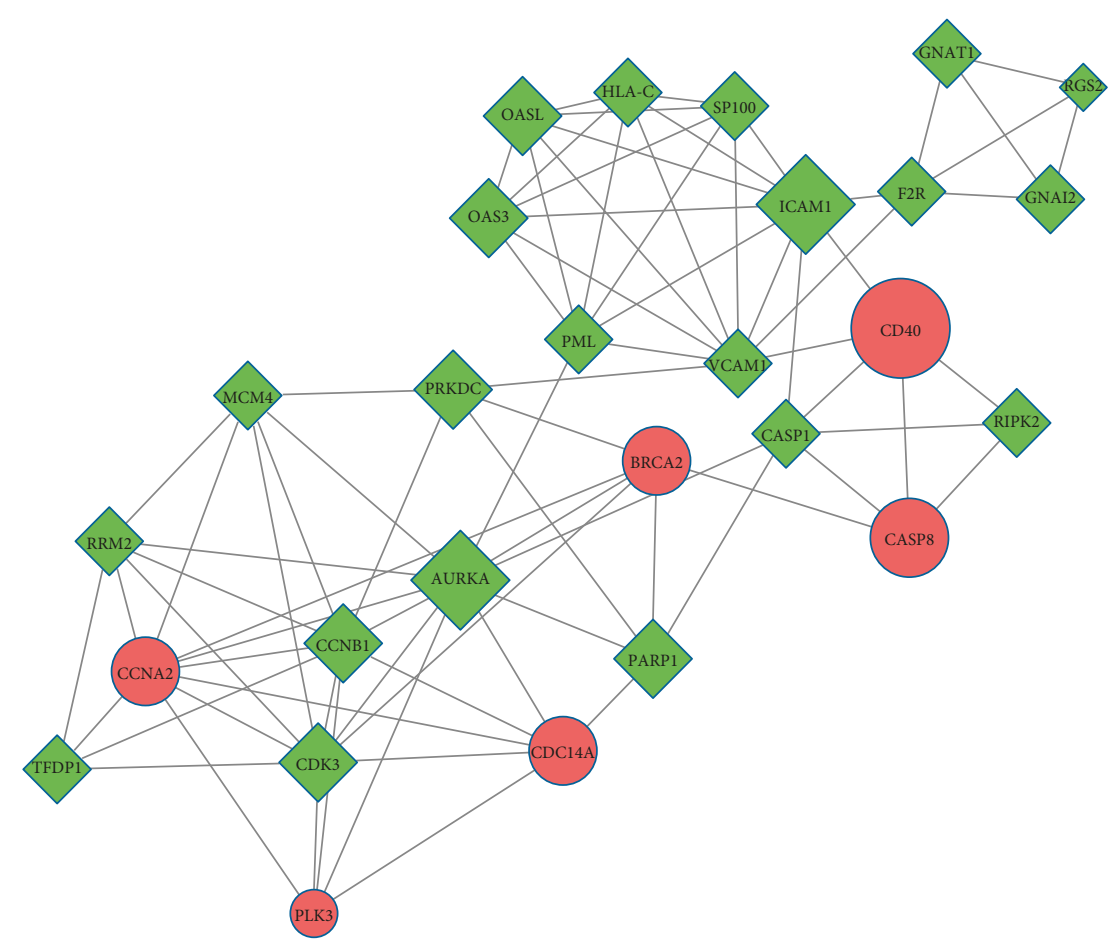

(c)

FiguRE 4: Subnetworks of differentially expressed genes (DEGs). (a) Subnetwork a; (b) subnetwork b; (c) subnetwork c. Red circles represent upregulated DEGs, and green diamonds represent downregulated DEGs.

\section{Results}

3.1. Analysis ofDEGs. The boxplot of the preprocessed data indicated good normalization (Figure 1). In total, 22,277 probes were obtained, among which 971 probes were differentially expressed. After annotation, 819 DEGs in patients with PD compared with those in healthy matched controls were identified (Supplementary Table 1), including 359 upregulated DEGs and 460 downregulated DEGs.
3.2. Pathway Enrichment Analysis. GO and KEGG pathway enrichment analyses for the up- and downregulated DEGs were performed (Supplementary Table 2). The significant GO terms and KEGG pathways are shown in Figure 2. The upregulated DEGs were significantly enriched in four KEGG pathways, namely, metabolic pathways, inositol phosphate metabolism, mRNA surveillance pathway, and RNA degradation, and GO terms such as transcription, DNAtemplate processing, and rRNA processing (Figure 2(a)). 
TABLE 2: List of KEGG pathways of subnetworks.

\begin{tabular}{lccccc}
\hline \multirow{2}{*}{ Subnetwork } & $\begin{array}{c}\text { Pathway } \\
\text { ID }\end{array}$ & Pathway name & Count & $p$ value & Genes \\
\hline \multirow{2}{*}{ Subnetwork } & hsa04080 & Neuroactive ligand-receptor interaction & 5 & $1.40 E-04$ & MCHR1, PTGER3, S1PR5, FPR3, NPY1R \\
a & hsa04062 & Chemokine signaling pathway & 3 & $1.80 E-02$ & CCR9, CCR6, CCR4 \\
& hsa04060 & Cytokine-cytokine receptor interaction & 3 & $2.74 E-02$ & CCR9, CCR6, CCR4 \\
& hsa04110 & Cell cycle & 6 & $1.31 E-04$ & CCNB1, CDC14A, PRKDC, CCNA2, MCM4, \\
& hsa05416 & Viral myocarditis & 4 & $1.62 E-03$ & TFDP1 \\
hsa05168 & Herpes simplex infection & 5 & $6.03 E-03$ & SP100, CASP8, OAS3, PML, HLA-C \\
hsa04514 & Cell adhesion molecules & 4 & $1.70 E-02$ & VCAM1, ICAM1, HLA-C, CD40 \\
c & Malaria & 3 & $1.78 E-02$ & VCAM1, ICAM1, CD40 \\
& hsa05144 & 3 & $2.00 E-02$ & CASP8, RIPK2, CASP1 \\
& hsa04621 & NOD-like receptor signaling pathway & 3 & $2.93 E-02$ & CCNB1, RRM2, CASP8 \\
& hsa05164 & p53 signaling pathway & Influenza A & $3.32 E-02$ & ICAM1, OAS3, PML, CASP1 \\
& hsa04914 & Progesterone-mediated oocyte & 3 & $4.30 E-02$ & CCNB1, GNAI2, CCNA2 \\
& hsa05169 & Epstein-Barr virus infection & 4 & $4.42 E-02$ & ICAM1, HLA-C, CD40, CCNA2 \\
& hsa05203 & Viral carcinogenesis & 4 & $4.60 E-02$ & SP100, CASP8, HLA-C, CCNA2 \\
& hsa04064 & NF-kappa B signaling pathway & 3 & $4.84 E-02$ & VCAM1, ICAM1, CD40 \\
\hline
\end{tabular}

KEGG, Kyoto Encyclopedia of Genes and Genomes.
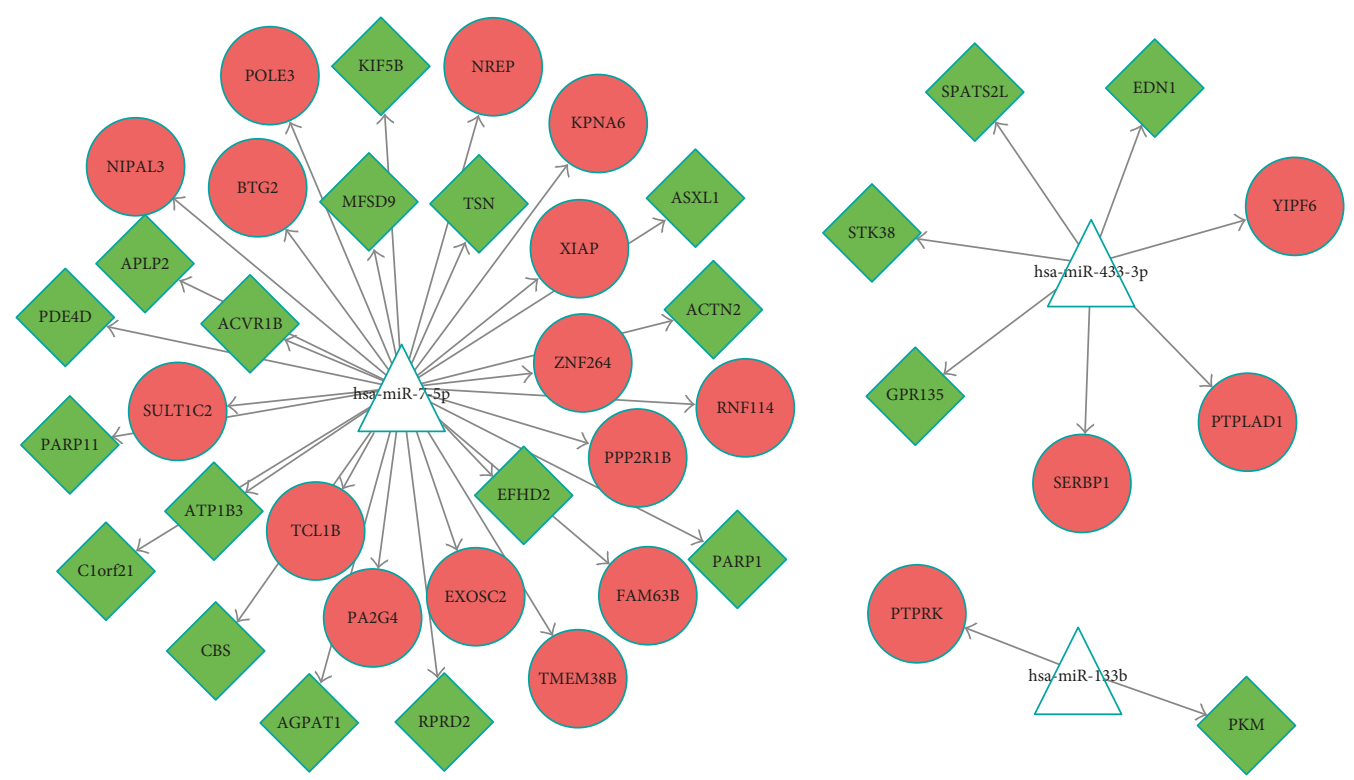

FiguRE 5: MicroRNA- (miRNA-) target regulatory networks of differentially expressed genes (DEGs). Triangles represent miRNAs, red circles represent upregulated DEGs, and green diamonds represent downregulated DEGs.

The downregulated DEGs were enriched in pathways such as those of influenza A, viral myocarditis, and TNF signaling and GO terms such as cytoplasm, cell surface, and interferon gamma-mediated signaling pathway (Figure 2(b)).

3.3. PPI Network Analysis. The PPI network, including 605 nodes and 1937 PPI pairs, is shown in Figure 3. The top 10 DEGs with the highest degree included five upregulated DEGs such as estrogen receptor 1 (ESR1), mechanistic target of rapamycin (MTOR), ATM serine/threonine kinase (ATM), CD40 molecule (CD40) and thymidine kinase 2, mitochondrial (TK2), and five downregulated DEGs such as mitogen-activated protein kinase 14 (MAPK14), phosphatase and tensin homolog $(P T E N)$, intercellular adhesion molecule 1 (ICAM1), aurora kinase A (AURKA), and protein kinase, DNA-activated, catalytic polypeptide (PRKDC) (Table 1). Three subnetworks were identified (subnetworks a-c). Subnetwork a (Figure 4(a)) included nine nodes and 36 PPI pairs, and these genes were significantly enriched in three KEGG pathways (Table 2), including neuroactive ligand-receptor interaction, chemokine signaling pathway, and cytokinecytokine receptor interaction. Subnetwork b (Figure 4(b)) included seven nodes and 21 PPI pairs, and these genes were not enriched in any KEGG pathway. Subnetwork c (Figure 4(c)) included 27 nodes and 81 PPI pairs, and these genes were enriched in 12 KEGG pathways (Table 2), such as cell cycle, herpes simplex infection, and NF- $\kappa$ B signaling pathways. 


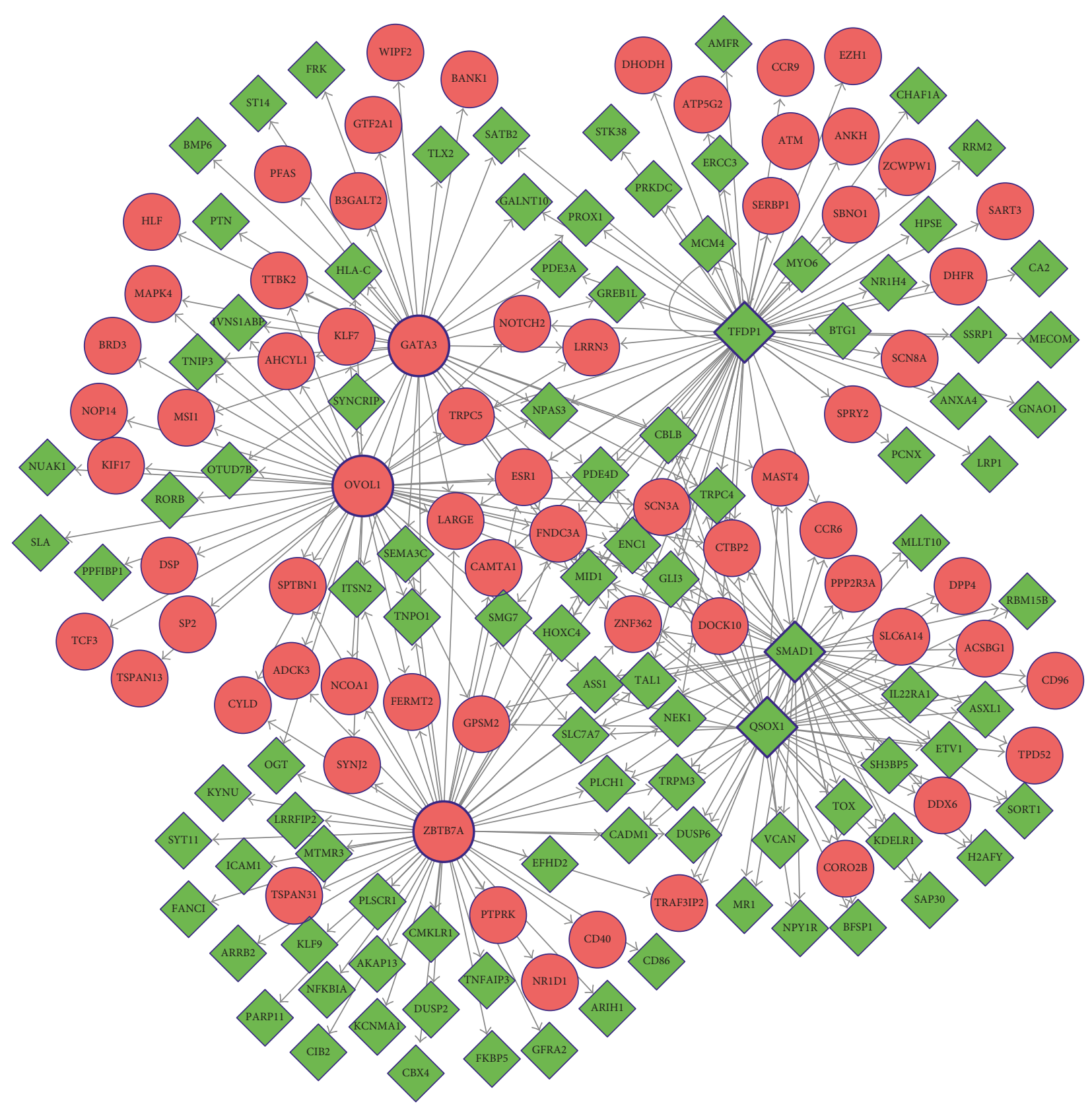

FIGURE 6: The TF-target regulatory network of differentially expressed genes (DEGs). Blue boxed figures represent TFs, red circles represent upregulated genes, and green diamonds represent downregulated genes. TF, transcription factor.

In addition, ICAM1 was involved in six KEGG pathways of subnetwork c, such as viral myocarditis, cell adhesion molecules (CAMs), and NF- $\kappa$ B signaling pathways (Table 2). The detailed information existed in PPI network, and three subnetworks are shown in Supplementary Table 3.

3.4. miRNA-Target Regulatory Network Analysis. According to the data from the miR2disease database, six miRNAs were identified to be associated with PD and 698 miRNA-gene pairs were obtained by searching miRWalk2.0. A total of 40 miRNA-target interaction pairs were obtained by comparing miRNA-gene pairs with DEGs, and subsequently, the
miRNA-target regulatory network was constructed. The network (Figure 5) contained 40 miRNA-target interaction pairs and 43 nodes (Supplementary Table 4), among which three miRNAs (hsa-miR-7-5p, hsa-miR-433-3p, and hsamiR-133b) were included.

3.5. TF-Target Regulatory Network Analysis. According the information of TF-target interaction databases such as Transfac, Jaspar, and Encode in the Cytoscape software, a total of 83 TFs were identified from the PPI network, forming 5371 TF-gene pairs. Among the 83 TFs, six were differentially expressed: three upregulated ones, that is, zinc 
TABLE 3: List of top 20 nodes with higher degree in transcription factor-target regulatory network.

\begin{tabular}{|c|c|c|c|}
\hline Gene & Full name & Description & Degree \\
\hline TFDP1* & Transcription factor $\mathrm{Dp}-1$ & Down & 62 \\
\hline ZBTB7A* & Zinc finger and BTB domain-containing 7A & Up & 55 \\
\hline OVOL1* & Ovo-like transcriptional repressor 1 & Up & 46 \\
\hline SMAD $1^{*}$ & SMAD family member 1 & Down & 45 \\
\hline QSOX1* & Quiescin sulfhydryl oxidase 1 & Down & 44 \\
\hline GATA3* & GATA-binding protein 3 & Up & 38 \\
\hline ENC1 & Ectodermal-neural cortex 1 & Down & 6 \\
\hline FNDC3A & Fibronectin type III domain-containing $3 \mathrm{~A}$ & Up & 6 \\
\hline MID1 & Midline 1 & Down & 6 \\
\hline PDE4D & Phosphodiesterase 4D & Down & 5 \\
\hline ZNF362 & Zinc finger protein 362 & Up & 5 \\
\hline CBLB & $\mathrm{Cbl}$ proto-oncogene $\mathrm{B}$ & Down & 4 \\
\hline LARGE & LARGE xylosyl- and glucuronyltransferase & Up & 4 \\
\hline TRPC4 & $\begin{array}{l}\text { Transient receptor potential cation channel subfamily } \\
\text { C member } 4\end{array}$ & Down & 4 \\
\hline СТВР2 & C-terminal binding protein 2 & Up & 4 \\
\hline GLI3 & GLI family zinc finger 3 & Down & 4 \\
\hline SCN3A & Sodium voltage-gated channel alpha subunit 3 & Up & 4 \\
\hline TAL1 & $\begin{array}{c}\text { TAL BHLH transcription factor } 1 \text {, erythroid } \\
\text { differentiation factor }\end{array}$ & Down & 4 \\
\hline LRRN3 & Leucine rich repeat neuronal 3 & Up & 3 \\
\hline MAST4 & $\begin{array}{l}\text { Microtubule-associated serine/threonine kinase } \\
\text { family member } 4\end{array}$ & Up & 3 \\
\hline
\end{tabular}

*Transcription factor.

finger and BTB domain-containing 7A (ZBTB7A), ovo-like transcriptional repressor 1 (OVOL1), and GATA-binding protein 3 , and three downregulated ones, that is, transcription factor dp-1 (TFDP1), SMAD family member 1 (SMAD1), and quiescin sulfhydryl oxidase 1 (QSOX1). The TF-target regulatory network (Figure 6) was constructed and included 166 nodes and 288 interaction pairs (Supplementary Table 5). The top 20 nodes with the highest degree are listed in Table 3, including the six TFs described above and 14 other DEGs, such as ectodermal-neural cortex 1 , fibronectin type III domain-containing 3A, and midline 1 , which were coregulated by the six TFs.

\section{Discussion}

$\mathrm{PD}$ is the second most common age-related neurodegenerative disease. However, the pathogenesis and genes involved in PD are not well known [42]. In this study, we performed a comprehensive bioinformatics analysis of the blood gene expression profile using the GSE72267 dataset. The results suggested that four key pathways (metabolic pathways, TNF signaling pathway, rRNA processing, and cytoplasm), the key gene ICAM1, three miRNAs (hsa-miR7-5p, hsa-miR-433-3p, and hsa-miR-133b), and six TFs (ZBTB7A, OVOL1, GATA3, TFDP1, SMAD1, and QSOX) might play important roles in $\mathrm{PD}$ development.

Our results revealed that the upregulated DEGs were enriched in the KEGG pathway "metabolic pathways" and the
GO term "rRNA processing," and the downregulated DEGs were enriched in the KEGG pathway "TNF signaling pathway" and the GO term "cytoplasm." A previous study [43] demonstrated that some metabolic patterns were altered in patients with advanced PD. Multiple metabolic pathways are also involved in PD [44], which supports our study results. Cytoplasmic inclusions are a pathological hallmark of PD [45]. Lewy body pathology is involved [46, 47], and glial cytoplasmic inclusions are associated with Lewy bodies [48]. Thus, the GO term "cytoplasm" may play a role in PD. Furthermore, TNF receptor-associated protein is excluded from the nucleolus and is sequestered to the cytoplasm by TNF receptor-associated factor 6 , thereby altering ribosomal RNA (rRNA) biogenesis [49]. The TNF signaling pathway is also involved in PD [50], and rRNA transcription is repressed in patients with PD [51]. Therefore, the GO term "rRNA processing" and the KEGG pathway "TNF signaling pathway" may play important roles in PD. Altogether, the metabolic pathways, TNF signaling pathway, rRNA processing, and cytoplasm are essentially involved in PD pathogenesis.

ICAM1 was among the top 10 DEGs in the PPI network. Moreover, ICAM1 gene was involved in six KEGG pathways for subnetwork c. ICAM1 is involved in the adhesion and transmigration of leukocytes across the endothelium, promoting brain inflammation and resulting in brain diseases [52]. T helper 17 cells can exert a neurotoxic effect in the brain parenchyma of patients with PD by interacting with ICAM1 and leukocyte function-associated antigen 1 [53]. In 
addition, ICAM1 is involved in persistent inflammation in PD [54]. Our results from the KEGG pathway analysis for genes in subnetworks revealed that ICAM1 might play roles in viral myocarditis and CAMs and thus contributed to PD.

The miRNA-target regulatory network analysis identified three miRNAs involved in PD, namely, hsa-miR-7-5p, hsamiR-433-3p, and hsa-miR-133b. A study described miR-7-2 dysregulation (the stem loop of hsa-miR-7-5p) in Parkinson's patient's leukocytes [55] and revealed that hsa-miR-7-5p expression decreased in PD, possibly upregulating $\alpha-S Y N$, a PD-related gene [56]. The variation of the hsa-miR-433- (the stem loop of hsa-miR-433-3p-) binding site of fibroblast growth factor 20 can lead to $\alpha-S Y N$ overexpression, increasing the risk for PD [57]. hsa-miR-133b expression is increased in the cerebrospinal fluid of patients with PD [58]; however, its expression levels in serum is decreased, which is related to low serum ceruloplasmin levels [59]. hsa-miR-133b is also deficient in the midbrain tissue of patients with $\mathrm{PD}$ and is associated with the maturation and function of midbrain dopaminergic neurons [60]. Notably, reduced circulating levels of miR-433 and miR-133b are considered as promising biomarkers for PD [61]. Therefore, we speculate that the three miRNAs, including hsa-miR-75p, hsa-miR-433-3p, and hsa-miR-133b may play important roles in PD.

TFs are important regulators of target gene expressions $[53,62]$. In this study, we analyzed DEGs in the PPI network to screen TFs involved in PD. Among the 83 TFs identified in the PPI network, six were found to be differentially expressed. ZBTB7A, OVOL1, and GATA3 were upregulated in patients with $\mathrm{PD}$ compared with those in healthy matched controls, whereas TFDP1, SMAD1, and QSOX1 were downregulated. ZBTBZA is a tumor suppressor, which is involved in several cancers such as prostate and nonsmall cell lung cancers [63-65]. OVOL1, encoding a zinc finger protein, is expressed in embryonic epidermal progenitor cells and is an inducer of mesenchymal-to-epithelial transition in human cancers $[66,67]$. GATA3, a member of the GATA family, is a regulator of T-cell development and plays roles in endothelial cells $[68,69]$. TFDP1 is involved in the cell cycle and contributes to hepatocellular carcinomas [70, 71], SMAD1 is involved in multiple pathways [72, 73], and QSOX1 plays roles in some cancers such as breast cancer and neuroblastoma [74-76]. However, there are few reports regarding the involvement of these TFs in PD. Hence, further studies regarding the associations between the TFs identified in this study and PD are warranted.

In conclusion, our data demonstrated that the metabolic pathways, TNF signaling pathway, rRNA processing, and cytoplasm play important roles in PD pathogenesis; ICAM1 might also play a vital role. Besides six TFs, three miRNAs, including hsa-miR-7-5p, hsa-miR-433-3p, and hsa-miR$133 \mathrm{~b}$, may be involved in PD. However, because of the study limitations, further investigation remains to be performed in the future.

\section{Conflicts of Interest}

The authors declare that there are no conflicts of interest regarding the publication of this article.

\section{Acknowledgments}

This study was supported by the Construction of Accurate Technology Innovation Centers of Nervous System Disease, Jilin Province (no. 20170623006TC), and Study on the Mechanism of the Parkinson lncrna, Jilin Provincial Department of Finance Project.

\section{Supplementary Materials}

Supplementary 1. Table 1: all up- and downregulated differentially expressed genes.

Supplementary 2. Table 2: GO and KEGG pathway enrichment analyses for the up- and downregulated differentially expressed genes. GO, Gene Ontology; KEGG, Kyoto Encyclopedia of Genes and Genomes.

Supplementary 3. Table 3: the detailed information existing in protein-protein interaction (PPI) network and three subnetworks.

Supplementary 4. Table 4: the detailed information about the miRNA-target regulatory network.

Supplementary 5. Table 5: the detailed information about transcription factor- (TF-) target regulatory network.

\section{References}

[1] W. Dauer and S. Przedborski, "Parkinson's disease: mechanisms and models," Neuron, vol. 39, no. 6, pp. 889-909, 2003.

[2] A. E. Lang and A. M. Lozano, "Parkinson's disease. Second of two parts," New England Journal of Medicine, vol. 339, no. 16, pp. 1130-1143, 1998.

[3] A. E. Lang and A. M. Lozano, "Parkinson's disease," New England Journal of Medicine, vol. 37, p. 198, 1998.

[4] L. V. Kalia and A. E. Lang, "Parkinson's disease," Lancet, vol. 386, no. 9996, pp. 896-912, 2015.

[5] D. J. Moore, A. B. West, V. L. Dawson, and T. M. Dawson, "Molecular pathophysiology of Parkinson's disease," Annual Review of Neuroscience, vol. 28, no. 1, pp. 57-87, 2005.

[6] S. K. Bae, C. H. Heo, D. J. Choi et al., "A ratiometric twophoton fluorescent probe reveals reduction in mitochondrial $\mathrm{H} 2 \mathrm{~S}$ production in Parkinson's disease gene knockout astrocytes," Journal of the American Chemical Society, vol. 135, no. 26, pp. 9915-9923, 2013.

[7] R. Calligaris, M. Banica, P. Roncaglia et al., "Blood transcriptomics of drug-naïve sporadic Parkinson's disease patients," BMC Genomics, vol. 16, no. 1, pp. 1-14, 2015.

[8] L. Gong, Q. L. Zhang, N. Zhang et al., "Neuroprotection by urate on 6-OHDA-lesioned rat model of Parkinson's disease: linking to Akt/GSK3 $\beta$ signaling pathway," Journal of Neurochemistry, vol. 123, no. 5, pp. 876-885, 2012.

[9] K. U. Tufekci, E. C. Bayin, S. Genc, and K. Genc, "The Nrf2/ARE pathway: a promising target to counteract mitochondrial dysfunction in Parkinson's disease," Parkinson's Disease, vol. 2011, Article ID 314082, 14 pages, 2011. 
[10] D. Cheng, A. M. Jenner, G. Shui et al., "Lipid pathway alterations in Parkinson's disease primary visual cortex," PLoS One, vol. 6, Article ID e17299, 2011.

[11] R. Deumens, A. Blokland, and J. Prickaerts, "Modeling Parkinson's disease in rats: an evaluation of 6-OHDA lesions of the nigrostriatal pathway," Experimental Neurology, vol. 175, no. 2, pp. 303-317, 2002.

[12] E. Leroy, R. Boyer, G. Auburger et al., "The ubiquitin pathway in Parkinson's disease," Nature, vol. 395, no. 6701, pp. 451-452, 1998.

[13] T. Pan, S. Kondo, W. Le, and J. Jankovic, "The role of autophagy-lysosome pathway in neurodegeneration associated with Parkinson's disease," Brain, vol. 131, no. 8, pp. 1969-1978, 2008.

[14] R. M. Perrett, Z. Alexopoulou, and G. K. Tofaris, "The endosomal pathway in Parkinson's disease," Molecular \& Cellular Neurosciences, vol. 66, pp. 21-28, 2015.

[15] A. L. Whone, R. Y. Moore, P. P. Piccini, and D. J. Brooks, "Plasticity of the nigropallidal pathway in Parkinson's disease," Annals of Neurology, vol. 53, no. 2, pp. 206-213, 2003.

[16] B. D. Cholewa, X. Liu, and N. Ahmad, "The role of polo-like kinase 1 in carcinogenesis: cause or consequence?," Cancer Research, vol. 73, no. 23, pp. 6848-6855, 2013.

[17] E. Miñonesmoyano, S. Porta, G. Escaramís et al., "MicroRNA profiling of Parkinson's disease brains identifies early downregulation of miR-34b/c which modulate mitochondrial function," Human Molecular Genetics, vol. 20, no. 15, p. 3067, 2011.

[18] M. L. De, E. Coto, L. F. Cardo et al., "Analysis of the MicroRNA-133 and PITX3 genes in Parkinson's disease," American Journal of Medical Genetics Part B Neuropsychiatric genetics: The Official Publication of the International Society of Psychiatric Genetics, vol. 153B, no. 6, pp. 1234-1239, 2010.

[19] S. Li, X. Lv, K. Zhai et al., "MicroRNA-7 inhibits neuronal apoptosis in a cellular Parkinson's disease model by targeting Bax and Sirt2," American Journal of Translational Research, vol. 8, no. 2, pp. 993-1004, 2016.

[20] Y. Zhou, M. Lu, R. H. Du et al., "MicroRNA-7 targets Nodlike receptor protein 3 inflammasome to modulate neuroinflammation in the pathogenesis of Parkinson's disease," Molecular Neurodegeneration, vol. 11, no. 1, p. 28, 2016.

[21] W. Le, D. Nguyen, X. W. Lin et al., "Transcription factor PITX3 gene in Parkinson's disease," Neurobiology of Aging, vol. 32, no. 4, pp. 750-753, 2011.

[22] J. Li, J. A. Dani, and W. Le, "The role of transcription factor Pitx3 in dopamine neuron development and Parkinson's disease," Current Topics in Medicinal Chemistry, vol. 9, no. 10, pp. 855-859, 2009.

[23] X. Xie, H. Liu, and Y. Gao, "Expression changes of transcription factor EN1 in the midbrain of mice model of Parkinson's disease," Chinese Journal of Rehabilitation Medicine, vol. 27, pp. 197-200, 2012.

[24] A. Cuadrado, P. Morenomurciano, and J. Pedrazachaverri, "The transcription factor Nrf2 as a new therapeutic target in Parkinson's disease," Expert Opinion on Therapeutic Targets, vol. 13, no. 3, pp. 319-329, 2009.

[25] P. M. Flood, L. Qian, L. J. Peterson et al., "Transcriptional factor NF- $\kappa \mathrm{B}$ as a target for therapy in Parkinson's disease," Parkinson's Disease, vol. 2011, Article ID 216298, 8 pages, 2011.

[26] M. Kurzawski, M. Białecka, J. Sławek, G. Kłodowskaduda, and M. Droździk, "Association study of GATA-2 transcription factor gene (GATA2) polymorphism and Parkinson's disease," Parkinsonism \& Related Disorders, vol. 16, no. 4, pp. 284-287, 2009.
[27] N. V. Soshnikova, N. E. Vorob’Eva, A. A. Kolacheva et al., "Ratio of transcription factor PHF10 splice variants in lymphocytes as a molecular marker of Parkinson's disease," Molecular Biology, vol. 50, no. 4, pp. 695-702, 2016.

[28] T. Barrett, T. O. Suzek, D. B. Troup et al., "NCBI GEO: mining millions of expression profiles-database and tools," Nucleic Acids Research, vol. 33, pp. D562-D566, 2005.

[29] L. Gautier, L. Cope, B. M. Bolstad, and R. A. Irizarry, "affy-analysis of Affymetrix GeneChip data at the probe level," Bioinformatics, vol. 20, no. 3, pp. 307-315, 2004.

[30] G. Smyth and G. K. Smyth, "Limma: linear models for microarray data," Bioinformatics and Computational Biology Solution Using $R$ and Bioconductor, Springer, Berlin, Germany, 2005.

[31] I. Hulsegge, A. Kommadath, and M. A. Smits, "Globaltest and GOEAST: two different approaches for Gene Ontology analysis," BMC Proceedings, vol. 3, no. 4, p. S10, 2009.

[32] T. G. O. Consortium, M. Ashburner, C. A. Ball et al., "Gene ontology: tool for the unification of biology," Nature Genetics, vol. 25 , no. 1, pp. 25-29, 2000.

[33] M. Kanehisa and S. Goto, "KEGG: Kyoto Encyclopedia of Genes and Genomes," Nucleic Acids Research, vol. 27, no. 1, pp. 27-30, 1999.

[34] D. W. Huang, B. T. Sherman, and R. A. Lempicki, "Systematic and integrative analysis of large gene lists using DAVID bioinformatics resources," Nature Protocol, vol. 4, no. 1, pp. 44-57, 2009.

[35] D. Szklarczyk, A. Franceschini, S. Wyder et al., "STRING v10: protein-protein interaction networks, integrated over the tree of life," Nucleic Acids Research, vol. 43, pp. D1-D447, 2015.

[36] P. Shannon, A. Markiel, O. Ozier et al., "Cytoscape: a software environment for integrated models of biomolecular interaction networks," Genome Research, vol. 13, no. 11, pp. 2498-2504, 2003.

[37] Y. Tang, M. Li, J. Wang, Y. Pan, and F. X. Wu, "CytoNCA: a cytoscape plugin for centrality analysis and evaluation of protein interaction networks," Bio Systems, vol. 127, pp. 6772, 2014.

[38] W. P. Bandettini, P. Kellman, C. Mancini et al., "MultiContrast Delayed Enhancement (MCODE) improves detection of subendocardial myocardial infarction by late gadolinium enhancement cardiovascular magnetic resonance: a clinical validation study," Journal of Cardiovascular Magnetic Resonance, vol. 14, no. 1, p. 83, 2012.

[39] Q. Jiang, Y. Wang, Y. Hao et al., "miR2Disease: a manually curated database for microRNA deregulation in human disease," Nucleic Acids Research, vol. 37, pp. D98-104, 2009.

[40] H. Dweep and N. Gretz, "miRWalk2.0: a comprehensive atlas of microRNA-target interactions," Nature Methods, vol. 12, no. 8, p. 697, 2015.

[41] R. Janky, A. Verfaillie, H. Imrichová et al., "iRegulon: from a gene list to a gene regulatory network using large motif and track collections," Plos Computational Biology, vol. 10, no. 7, p. e1003731, 2014.

[42] Y. Feng, J. Jankovic, and Y. C. Wu, "Epigenetic mechanisms in Parkinson's disease," Journal of the Neurological Sciences, vol. 349, no. 1-2, pp. 3-9, 2015.

[43] M. G. Moreno, C. Sánchez, G. Vazquez, J. Altamirano, and M. Avilarodriguez, "Metabolic mismatch patterns in patients with advanced Parkinson's disease on ${ }^{18} \mathrm{~F}$-FDOPA, ${ }^{11} \mathrm{C}$ Raclopride and ${ }^{11}$ C-DTBZ PET/CT," Journal of Nuclear Medicine, vol. 56, no. 3, p. 1890, 2015.

[44] M. Bonin, S. Poths, H. Osaka, YL. Wang, K. Wada, and O. Riess, "Microarray expression analysis of gad mice 
implicates involvement of Parkinson's disease associated UCH-L1 in multiple metabolic pathways," Molecular Brain Research, vol. 126, no. 1, pp. 88-97, 2004.

[45] M. Ihara, H. Tomimoto, H. Kitayama et al., "Association of the cytoskeletal GTP-binding protein Sept4/H5 with cytoplasmic inclusions found in Parkinson's disease and other synucleinopathies," Journal of Biological Chemistry, vol. 278, no. 26, pp. 24095-24102, 2003.

[46] K. A. Mills, Z. Mari, C. Bakker et al., "Gait function and locus coeruleus Lewy body pathology in 51 Parkinson's disease patients," Parkinsonism \& Related Disorders, vol. 33, pp. 102-106, 2016.

[47] Y. Saito, A. Shioya, T. Sano, H. Sumikura, M. Murata, and S. Murayama, "Lewy body pathology involves the olfactory cells in Parkinson's disease and related disorders," Movement Disorders Official Journal of the Movement Disorder Society, vol. 31, no. 1, pp. 135-138, 2016.

[48] A. Mochizuki, Y. Komatsuzaki, and S. I. Shoji, "Association of Lewy bodies and glial cytoplasmic inclusions in the brain of Parkinson's disease," Acta Neuropathologica, vol. 104, pp. 534-537, 2002.

[49] S. Vilotti, M. Codrich, M. D. Ferro et al., "Parkinson's disease DJ-1 L166P alters rRNA biogenesis by exclusion of TTRAP from the nucleolus and sequestration into cytoplasmic aggregates via TRAF6," PLoS One, vol. 7, no. 4, Article ID e35051, 2012.

[50] T. N. Martinez, "Neuroinflammation, TNF, and ceramide signaling: putative pathways for neurotoxicity in Parkinson's disease," Diss., 2010.

[51] H. Kang and J. H. Shin, "Repression of rRNA transcription by PARIS contributes to Parkinson's disease," Neurobiology of Disease, vol. 73, pp. 220-228, 2015.

[52] J. Y. Choi and S. A. Jo, "KDM7A histone demethylase mediates TNF- $\alpha$-induced ICAM1 protein upregulation by modulating lysosomal activity," Biochemical \& Biophysical Research Communications, vol. 478, no. 3, pp. 1355-1362, 2016.

[53] Q. Zhao, H. Liu, C. Yao, J. Shuai, and X. Sun, "Effect of dynamic interaction between microRNA and transcription factor on gene expression," Biomed Research International, vol. 2016, Article ID 2676282, 10 pages, 2016.

[54] J. Miklossy, D. D. Doudet, C. Schwab, S. Yu, E. G. Mcgeer, and P. L. Mcgeer, "Role of ICAM-1 in persisting inflammation in Parkinson disease and MPTP monkeys," Experimental Neurology, vol. 197, no. 2, pp. 275-283, 2006.

[55] L. Soreq, M. Bronstein, D. S. Greenberg et al., "Small RNA sequencing-microarray analyses in Parkinson leukocytes reveal deep brain stimulation-induced splicing changes that classify brain region transcriptomes," Frontiers in Molecular Neuroscience, vol. 6, p. 10, 2013.

[56] E. Junn, K. W. Lee, B. S. Jeong, T. W. Chan, J. Y. Im, and M. M. Mouradian, "Repression of alpha-synuclein expression and toxicity by microRNA-7," Proceedings of the National Academy of Sciences of the United States of America, vol. 106, no. 31, pp. 13052-13507, 2009.

[57] G. Wang, J. van der Walt, Y. Li et al., "Variation in the miRNA-433 binding site of FGF20 confers risk for Parkinson disease by overexpression of alpha-synuclein," American Journal of Human Genetics, vol. 82, no. 2, pp. 283-289, 2008.

[58] X. Ma, J. Ren, Y. Jiao, J. Yang, F. Xu, and Y. Song, "Expression of miR-133b and its clinical significance in cerebrospinal fluid of patients with Parkinson's disease," Modern Journal of Integrated Traditional Chinese \& Western Medicine, vol. 23, no. 24, pp. 2656-2658, 2014.
[59] N. Zhao, L. Jin, G. Fei, Z. Zheng, and C. Zhong, "Serum microRNA-133b is associated with low ceruloplasmin levels in Parkinson's disease," Parkinsonism \& Related Disorders, vol. 20, no. 11, pp. 1177-1180, 2014.

[60] J. Kim, K. Inoue, J. Ishii et al., "A MicroRNA feedback circuit in midbrain dopamine neurons," Science, vol. 317, no. 5842, pp. 1220-1224, 2007.

[61] X. Zhang, R. Yang, B. L. Hu et al., "Reduced circulating levels of miR-433 and miR-133b are potential biomarkers for Parkinson's disease," Frontiers in Cellular Neuroscience, vol. 11, p. 170, 2017.

[62] N. J. Martinez and A. J. Walhout, "The interplay between transcription factors and microRNAs in genome-scale regulatory networks," Bioessays, vol. 31, no. 4, pp. 435-445, 2009.

[63] K. Apostolopoulou, I. S. Pateras, K. Evangelou et al., "Gene amplification is a relatively frequent event leading to ZBTB7A (Pokemon) overexpression in non-small cell lung cancer," Journal of Pathology, vol. 213, no. 3, pp. 294-302, 2007.

[64] X. S. Liu, J. E. Haines, E. K. Mehanna et al., "ZBTB7A acts as a tumor suppressor through the transcriptional repression of glycolysis," Genes \& Development, vol. 28, no. 17, pp. 19171928, 2014.

[65] G. Wang, A. Lunardi, J. Zhang et al., "Zbtb7a suppresses prostate cancer through repression of a Sox9-dependent pathway for cellular senescence bypass and tumor invasion," Nature Genetics, vol. 45, no. 7, pp. 739-746, 2013.

[66] M. Nair, A. Teng, V. Bilanchone, A. Agrawal, B. Li, and X. Dai, "Ovoll regulates the growth arrest of embryonic epidermal progenitor cells and represses c-myc transcription," Journal of Cell Biology, vol. 173, no. 2, pp. 253-264, 2006.

[67] H. Roca, J. Hernandez, S. Weidner et al., "Transcription factors OVOL1 and OVOL2 induce the mesenchymal to epithelial transition in human cancer," PLoS One, vol. 8, no. 10, Article ID e76773, 2013.

[68] I. C. Ho, T. S. Tai, and S. Y. Pai, "GATA3 and the T-cell lineage: essential functions before and after T-helper-2-cell differentiation," Nature Reviews Immunology, vol. 9, no. 2, pp. 125-135, 2009.

[69] H. Song, J. Suehiro, Y. Kanki et al., "Critical role for GATA3 in mediating Tie2 expression and function in large vessel endothelial cells," Journal of Biological Chemistry, vol. 284, no. 42, pp. 29109-29124, 2009.

[70] X. Lu, X. D. Lv, Y. H. Ren et al., "Dysregulation of TFDP1 and of the cell cycle pathway in high-grade glioblastoma multiforme: a bioinformatic analysis," Genetics \& Molecular Research, vol. 15, no. 2, 2016.

[71] K. Yasui, H. Okamoto, S. Arii, and J. Inazawa, "Association of over-expressed TFDP1 with progression of hepatocellular carcinomas," Journal of Human Genetics, vol. 48, no. 12, pp. 609-613, 2003.

[72] LC. Fuentealba, E. Eivers, A. Ikeda et al., "Integrating patterning signals: Wnt/GSK3 regulates the duration of the BMP/Smad1 signal," Cell, vol. 131, no. 5, pp. 980-993, 2007.

[73] M. MaćiAssilva, P. A. Hoodless, S. J. Tang, M. Buchwald, and J. L. Wrana, "Specific activation of Smad1 signaling pathways by the BMP7 type I receptor, ALK2," Journal of Biological Chemistry, vol. 273, no. 40, pp. 25628-25636, 1998.

[74] D. Araújo and L. Nakao, "Expression level of quiescin sulfhydryl oxidase 1 (QSOX1) in neuroblastomas," European Journal of Histochemistry, vol. 58, no. 1, p. 2228, 2014.

[75] D. F. Lake and D. O. Faigel, "The emerging role of QSOX1 in cancer," Antioxid Redox Signal, vol. 21, no. 3, pp. 485-496, 2014. 
[76] N. Pernodet, F. Hermetet, P. Adami et al., "High expression of QSOX1 reduces tumorogenesis, and is associated with a better outcome for breast cancer patients," Breast Cancer Research, vol. 14, no. 5, p. R136, 2012. 


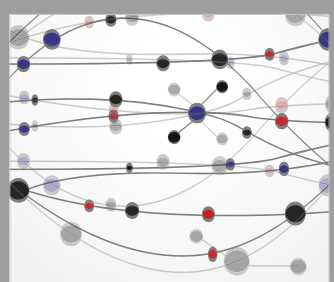

The Scientific World Journal
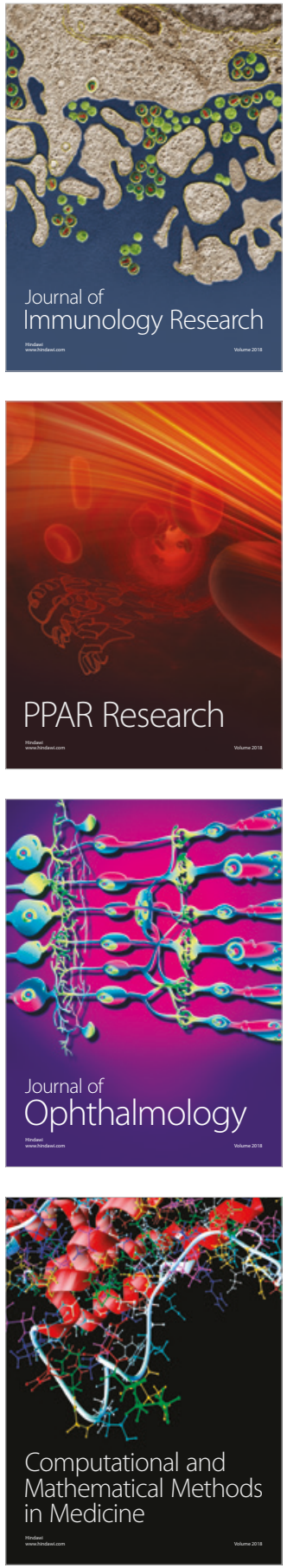

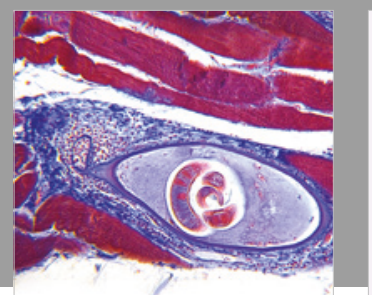

Gastroenterology Research and Practice

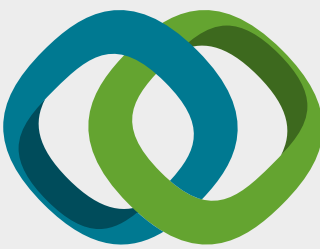

\section{Hindawi}

Submit your manuscripts at

www.hindawi.com
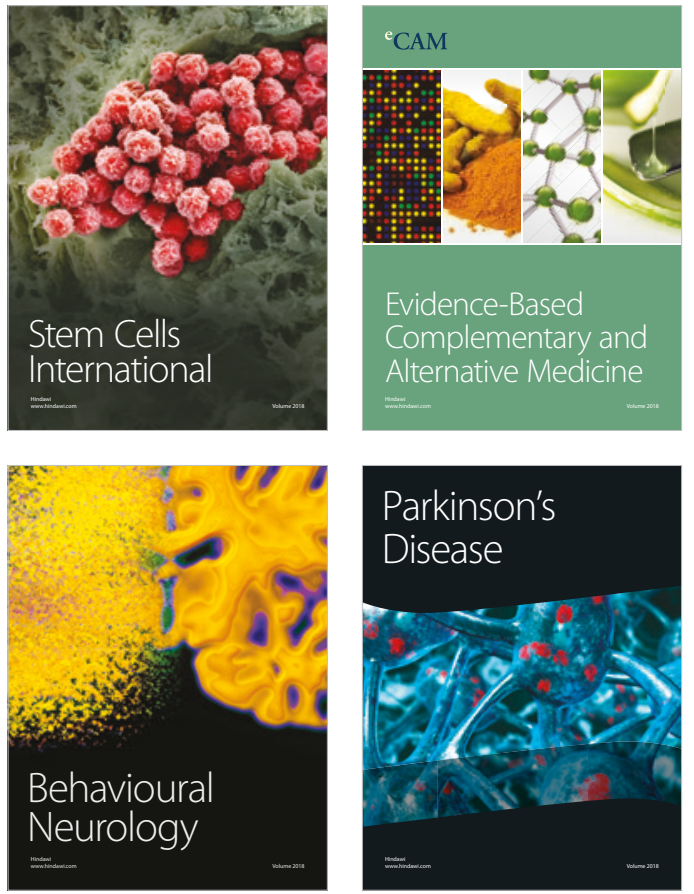

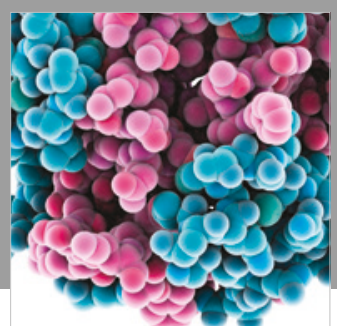

ournal of

Diabetes Research

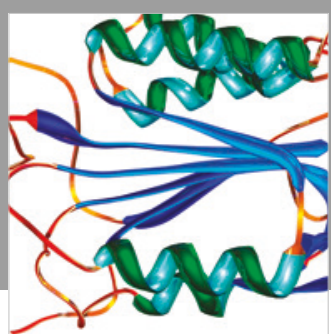

Disease Markers
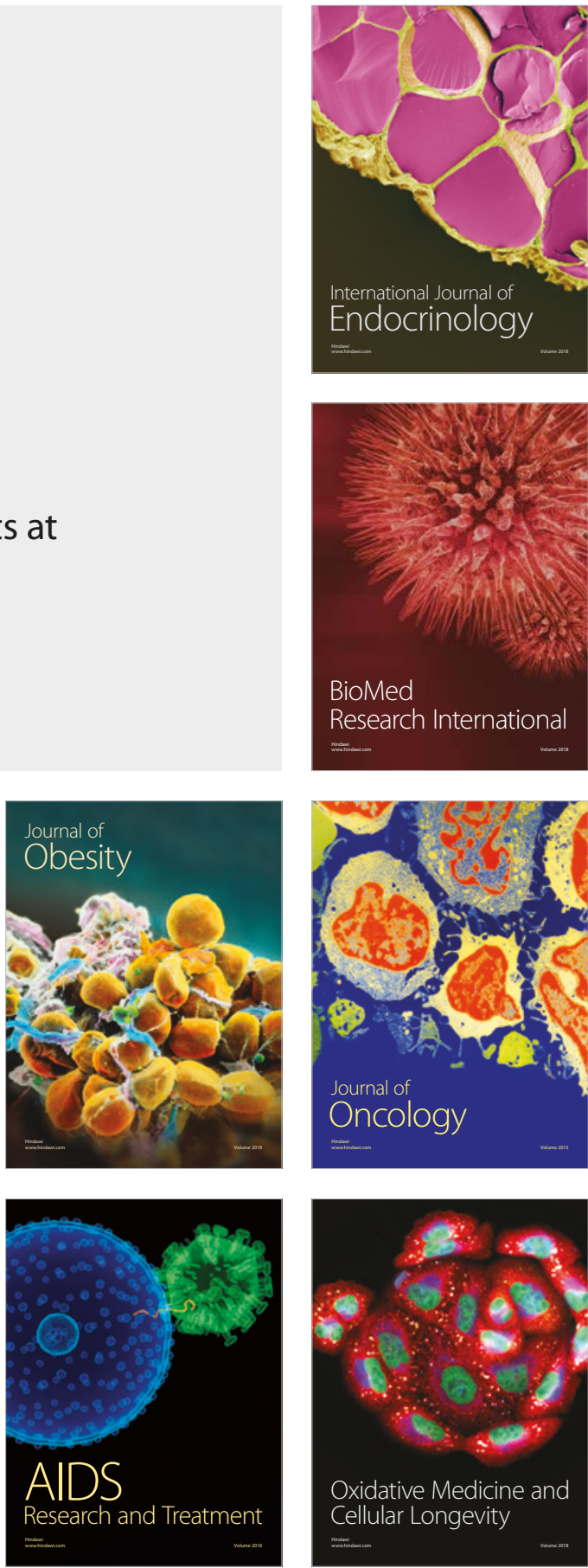